\title{
Toward a Concept of Population Balance Considering Age- Structure, Human Capital, and Intergenerational Equity
}

Wolfgang Lutz (lutz@iiasa.ac.at)

Warren Sanderson (sanders@iiasa.ac.at)

\section{Approved by}

Arne Jernelöv (jernelov@iiasa.ac.at)

Acting Director

January 12, 2001

Interim Reports on work of the International Institute for Applied Systems Analysis receive only limited review. Views or opinions expressed herein do not necessarily represent those of the Institute, its National Member Organizations, or other organizations supporting the work. 


\section{Contents}

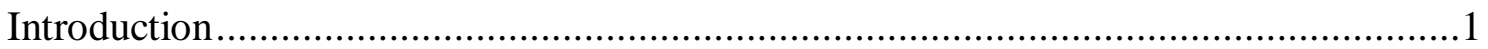

The International Political Dimension: Trying to Link Concerns about Population Growth, Aging, Education and the Relationship between Generations ..........................2

The Dynamics of Changing Age Structures: Analytical Approaches and Empirical

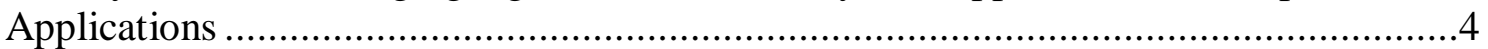

A First Attempt to Simulate Age, Cohort and Human Capital Aspects of "Population

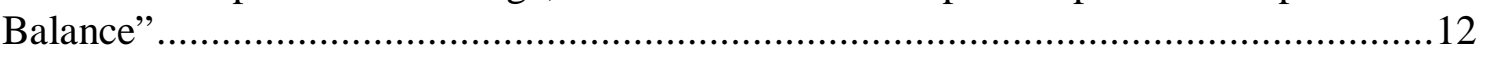

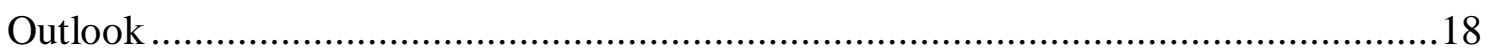

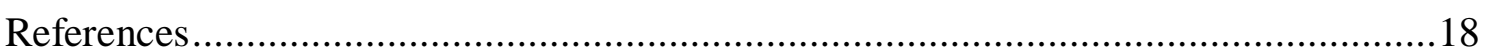




\section{Abstract}

This paper is a first step in trying to develop a concept incorporating population growth and population aging concerns under one analytical umbrella. Our approach is to try to achieve this through explicitly considering age structural changes through the analysis of age-specific growth rates and the new measure of Cohort Succession Ratios (CSR), which is introduced in this paper. In order to also address the issue of intergenerational equity on both the population aging and the sustainable development concerns, additional emphasis is put on human capital formation as measured by educational attainment as a proxy for productivity. Since age-, sex- and education-specific analysis can readily be carried out in a multi-state population projection context, this approach presents a feasible and operational strategy to work towards the goal of such a more general framework tentatively entitled "population balance."

The paper first discusses the political need for such a more general concept, and then works towards its operational definition through analytical and empirical considerations and the development of a simple simulation model considering age-, cohort- and education-specific productivity and income. 


\section{Acknowledgments}

This paper was presented at the IUSSP/APN Meeting on Age Structural Transitions and Policy Implications, held in Phuket, Thailand, November 8-10, 2000. 


\title{
Toward a Concept of Population Balance Considering Age- Structure, Human Capital, and Intergenerational Equity
}

\author{
Wolfgang Lutz and Warren Sanderson
}

\section{Introduction}

This paper consists of three parts that may at first sight look like three independent contributions. But coming from different sides and using very different tools they all work towards the common goal of trying to define a new concept tentatively labeled population balance. The ambitious task is to develop a paradigm that can cover both population growth and population aging concerns. In doing so it must consider both the role of the population variable in development and environmental change, and its role with respect to intergenerational equity. To achieve the bridging between population growth and aging concerns, we must first transcend the conventional focus on total growth rates and look at age-specific growth rates and other age-structural features. Secondly, we must transcend the realm of strictly demographic variables and endogenize into our model another individually measurable population characteristic which probably comes close to age as a key source of population heterogeneity, namely, level of education. The explicit consideration of human capital will allow us to link the implications of both population growth and aging under a joint umbrella framework.

The concept of population balance is still rather tentative and needs to be much more rigorously defined. This paper is only a first step in this direction. It is an attempt to approach the issue

(1) First, from the perspective of political needs and priorities: Why do we need a concept of that sort?

(2) Next, it will be approached from a measurement and empirical perspective: How can demographers measure age-structural change? In this context the definition of a new measure of Cohort Succession Ratios (CSRs) seems to be useful.

(3) Finally, it will be approached through a simple simulation model considering age-, cohort- and education-specific productivity and income.

Before we go into the political dimension of this concept and later into the analytical and empirical aspects, it is worth spending a few thoughts on the way the words "population" and "balance" are used here. Population is used in its original macro-level meaning describing a well-defined group of human beings with emphasis on quantitative aspects, which are primarily total size and growth rate, but also on changing distribution by age and sex and place of residence. There are other populationbased characteristics, such as marital status, labor force participation, ethnicity, educational status, etc., which do not represent core demographic dimensions, but often 
enrich population analysis in a meaningful way, especially if they can unambiguously be defined and measured as individual characteristics constituting sub-groups of the population. To such sub-populations the demographic tools of multi-state cohortcomponent analysis and projection can be readily applied. In the context of population balance, educational attainment is considered an additional population dimension because of its paramount importance for both the determinants and consequences of population change. Education is not only one of the most important sources of population heterogeneity (Lutz et al. 1999) but can also be readily measured and projected for all parts of the world (see Lutz and Goujon 2001). Hence the population dynamics considered in this paper comprises the population by age, sex, and educational attainment. In other settings population balance may also include other aspects of population, such as urban/rural place of residence or ethnicity.

The word "balance" does not have any well-defined meaning in population studies. In economics it is sometimes used in the sense of a dynamic consistency of certain elements of economic structure. In this context, "balance" should also have a highly dynamic meaning, like walking on a log in the water being exposed to all kinds of currents and winds and trying not to bend too far to either side and fall into the water. More generally, this assumes that a "balance" is better than an "imbalance" and implies a longer and better life for everybody concerned. "Population balance" then applies this to all cohorts (present and future), both sexes, different social groups and different regional (national) populations.

If, in a political sense, there is a potential model for the notion of "population balance" then it is the notion of "sustainable development." Although it is very poorly defined and people interpret it in surprisingly different ways, it still has become a politically extremely powerful notion ${ }^{1}$; the whole UN system is currently being restructured under the notion of sustainable development. While we hope to define "population balance" in a more precise and operational way than "sustainable development," it is also worth noting that the notion may benefit from not being too narrowly defined. Different people concerned with different aspects of the issue can read different things into it. It could mean the balance between population and the environment; it could mean a balance in the sex and age distribution, a balance across generations, etc. Hence, the challenge is to define the notion wide enough to make it relevant for different groups, but at the same time to provide a choice of different precise definitions and an analytical toolbox that allows for rigorous scientific analysis.

\section{The International Political Dimension: Trying to Link Concerns about Population Growth, Aging, Education and the Relationship between Generations}

International population analysis and politics is in disarray these days. There seems to be some confusion about the role of the population variable in social and economic development and environmental change. After decades of strong concern and concerted action against the population explosion, recent news about the end of world population growth to be expected during the second half of this century and fertility declines that

\footnotetext{
${ }^{1}$ This is not necessarily surprising, if one thinks, e.g., of how many wars the extremely vague notion of freedom has caused.
} 
are more rapid than expected seem to make these concerns less relevant, even though in individual world regions very strong growth is still to be expected. In parallel the process leading up to the 1994 Cairo Conference on Population and Development has brought a strong shift of emphasis from macro-level population concerns to individual rights and reproductive health concerns, i.e., an almost exclusive focus on the individual welfare dimension. Macro-level population-related considerations, which also impact on individual welfare, but in a more indirect way, were not given much attention. As an institutional consequence, e.g., in many national development agencies, the population offices, if they still exist at all, have become subsections of departments of health.

The population issue has largely become a specific sub-concern of health. But there is clearly more to population than one aspect of human health, the age-structural aspects discussed in this seminar being a good example, population-environment interactions being another important one. In the famous "Cairo Consensus," the Cairo Programme of Action mentions some of these macro issues, but there is no convincing substantive link between the main focus on reproductive health and the macro-level consequences of fertility trends. One cannot help feeling that these macro concerns were simply added in an unconnected fashion in order to satisfy some of the people who "were still stuck in the population concerns of yesterday," as some delegates put it informally. Reproductive health and individual welfare more generally are clearly very important aspects of population, but there is an urgent need to substantively link them to the population related macro-level concerns.

Simultaneously, but completely unrelated to this "Cairo Concensus," concern about population aging grew in some of the developed countries, especially in those that have been experiencing fertility well below replacement for several decades. This macro-level population concern tends to concentrate mainly on the sustainability of existing pension schemes, but also relates to expected impacts on productivity and international competitiveness and more recently on the need to rethink immigration policies. There is a rapidly increasing scientific literature and a rapidly increasing international concern. Yet in international fora the aging concerns tend to be treated in a completely unrelated way to the population growth and population-environment concerns and the individual-level reproductive health, female empowerment and freedom of choice concerns. There seems to be a need for some sort of an umbrella concept that links these two macro-level population concerns to each other and relates them to these individual-level concerns.

Another issue that gains prominence over the decades is that of intergenerational equity. Interestingly, this concern originally arose independently from all of the abovementioned population discussions although it is now clearly related to the concern about population aging. But the intergenerational dimension came to international political prominence through environmental concerns and the very definition of sustainable development (as given by the Brundlandt Commission). This definition is explicitly based on intergenerational concerns, i.e., improving living conditions without compromising the possibilities of future generations to meet their needs. More recently in the context of pension reforms in Europe that will become necessary due to expected population aging and the great dependence of pay-as-you-go pension systems on relative sizes of age groups, there is increasing concern over the fact that an everincreasing burden of the pension load is put on the shoulders of the younger and smaller cohorts. The degree to which the younger generations will be unduly hit by this 
depends, of course, also on the future course of productivity gains. This second factor shall be approximated by education in this paper.

Education and human capital formation have become another important force seen to influence both the consequences of rapid growth and rapid aging, because they are directly related to the productivity changes associated with changes in the age composition. Especially in the context of developing countries the World Bank has recently announced that education would be its top priority. But future productivity gains that are likely to be associated with higher technical skills are also seen as a possible solution to ameliorating the consequences of population aging. Because the educational composition of the total population and the educational enrollment rates of the young cohorts are not independent from age structural changes (e.g., if the youngest cohorts grow too rapidly, enrollment rates cannot keep pace) these two things should be viewed together. Hence both age structure and education, independently and in their interaction, play an important role in economic growth and consequently in intergenerational equity. The joint consideration of both may serve as a binding element between growth and aging concerns under a concept of "population balance."

\section{The Dynamics of Changing Age Structures: Analytical Approaches and Empirical Applications}

When talking about analytical approaches to describe and analyze age-distributional changes over time, fairly simultaneous but independent work by Sam Preston (Preston and Coale 1982; Horiuchi and Preston 1988) and Nathan Keyfitz (1987, 1990) comes to mind. In the following we will shortly discuss their approaches and give some empirical illustrations. We will also propose a new indicator tentatively called "cohort succession ratio" (CSR).

Following earlier work by Ansley Coale on a generalization of the stable population model to deal with variable growth rates, Horiuchi and Preston (1988) published a paper in Demography entitled "Age-specific Growth Rates: The Legacy of Past Population Dynamics." This paper demonstrates how the widely available but rarely used sets of age-specific growth rates in a population can be used to reconstruct all the pertinent features of a population's demographic history that are required to relate major demographic functions for a particular period to one another. They derive a formula,

$$
\ln \frac{N(a, t)}{n(a, t)}=-\int_{0}^{a} r(x, t) d x,
$$

which implies that the current age distribution $N(a, t)$ can be obtained by deriving the underlying stationary distribution $n(a, t)$ and converting it into the actual distribution by using age-specific growth rates, $r(x, t)$, at age $x$ and time $t$.

The density function of the stationary population is derived from current births and mortality schedules as given by

$$
n(a, t)=B(t) p(a, t),
$$

where $n(a, t)$ is the size that the cohort aged $a$ at time $t$ would attain if the cohort had the size $B(t)$ at birth and had mortality experiences as observed at time $t$. 
This concept has been used mainly to make estimates from incomplete data. But age-specific growth rates also provide useful information about the history of a population for periods during which vital rates were not recorded. Whether and how this approach can be used in addressing future population-related challenges needs further exploration.

Nathan Keyfitz's work on the issue essentially grew out of his empirical work in advising the Indonesian government on population-related challenges and the analysis of past and likely future world population trends. It may be best known under the keyword "international youth cohort" (Keyfitz 1990). This work focused both on the reconstruction of the onset of the post WWII population growth and on the possible consequences of age-structural discontinuities. In his 1987 paper entitled "The Demographic Discontinuity of the 1940s" he reconstructs the onset of the "population explosion" through the analysis of intercohort increases as given in more recent censuses. As an analytical tool Keyfitz mainly uses difference analysis (Keyfitz 1990). By describing the first and second differences between the sizes of subsequent cohorts in an often graphical manner, the demographic discontinuities become readily visible.

Although much of Keyfitz's work in this field is being used for the same purpose as that of Preston and others described above, namely the reconstruction and decomposition of past trends, Keyitz also extended his work into the future and makes projections of likely future discontinuities as embedded in the pattern of cohorts already born. Occasionally, he drew inferences beyond the limits of demography by, e.g., suggesting that during the mid-1990s Indonesia may see some major political shake up due to the numerous rather well-educated youth cohort entering the labor market (Keyfitz 1988). This bold forecast turned out to be quite accurate.

In studying intercohort changes, whether it is done through age-specific growth rates or differences between cohort sizes, both Preston and Keyfitz limit their focus to the comparison of adjacent cohorts. In the broader context of studying the implications of population aging and issues of intergenerational equity, it is useful to apply a more general scheme that generally describes the relationship between any two cohorts at a given time. To do this we introduce the notion of cohort succession ratios.

A cohort succession ratio can be defined as follows,

$$
\operatorname{CSR}(a, x, t)=N(a, t) / N(a+x, t)
$$

with $N$ standing for total population (of both sexes) and the indices $a$ for age (indicating either single or five-year age groups), $t$ for time, and $x$ for the age difference in years for the cohorts to be compared.

In the case of $x=1$ (or 5 in the case of five-year age groups) the pattern of CSRs should be very similar to that of age-specific growth rates and first difference because all compare adjacent cohorts with only the metric being different.

Figures 1a-e give empirical illustrations of the patterns of the different measures discussed over age and time for China. The data are five-year age groups from 1950 to 2050 as given by the UN (1999) estimates and projections. Figure 1a simply gives a 3-D plot of the total population of China (without Hongkong) for men and women combined. It shows that in 1950 China had a very young age distribution with some scars of history. Up to the 1970s the population at all age groups (interrupted by the turmoil of the 1960s) grew sharply with the movement along cohort lines clearly visible 
as ridges on the graph. Mortality improvements are shown by the fact that the ridges become more horizontal over time, i.e., there is less attrition of cohorts over time. After that low fertility results in declining initial sizes of birth cohorts with strong echo effects from the adult age distribution.

The first differences (calculated as the differences in size between the same fiveyear age groups in year $t+5$ minus that in year $t$ ) are plotted in Figure 1b. They give an even more pronounced picture of the consequences of the fertility trends and the associated echo effects on the starting sizes of cohorts. After the period of high fertility in the 1950s, there was a short but steep cut around 1960 associated with the great leap formward (which with some imagination is also seen in terms of higher mortality through a different inclination of the ridge). After recovery of fertility, another steep decline followed during the late 1960s. Finally, during the 1970s the consequences of the strict one child per family law became evident. The picture is somewhat distorted due to the fact that it only looks at the trends of five-year age groups. The data base probably includes a lot of estimated and smoothed data, especially for the older cohorts. The graph of second differences (Figure 1c) shows the same pattern in an even more pronounced fashion.

Figures 1d and 1e give the age-specific growth rates and the cohort succession ratios for subsequent cohorts. As expected these two indicators show rather similar patterns. Since these measures operate on a relative scale and are more sensitive to data problems, especially in the older age groups with few members, these graphs are restricted to the population below age 50. One thing is very clear from the comparison of all these figures. The strong fluctuations in Chinese fertility during the past decades are likely to produce strong waves of echoes, even in the case of almost constant future fertility as assumed by the UN.

One strength of the CSRs, as compared to the other measures, is the possibility of comparing non-adjacent cohorts. Figures 2 to 4 present such indicators for China, Thailand and Singapore. One ratio compares the youngest cohort (age 0-4) to the cohort aged 25-29. Since this 25-year difference is close to the mean age of childbearing, the ratios can be roughly interpreted in the sense of a gross reproduction rate that to some degree adjusts for infant and child mortality, or a net reproduction rate not considering the mortality of ages 5-24. Before 1970 this ratio tends to be above two in all three countries, indicating that the size of the youngest age group was more than twice that of the age group 25-29. In all three countries fertility decline brings down the ratio thereafter. In Singapore, a level below 1.0 has already been reached in 1980. Singapore is special in the sense that immigration of young adults has a great influence on these ratios.

The other ratio plotted in Figures 2 to 4 is of those aged 20-24 (a typical age for entering the formal labor market) and those aged 60-64 (a typical age for retiring, at least in industrialized countries). Hence, as a rough approximation this ratio could possibly describe labor force replacement (those entering divided by those leaving). If this ratio is above 1.0, the labor force is growing. In all three countries we see a very strong growth in the labor force between 1950 and the present. As to the future, Singapore will first reach a situation in which the labor force replacement will fall below 1.0. In Thailand and China, this is only likely to happen after 2020. 


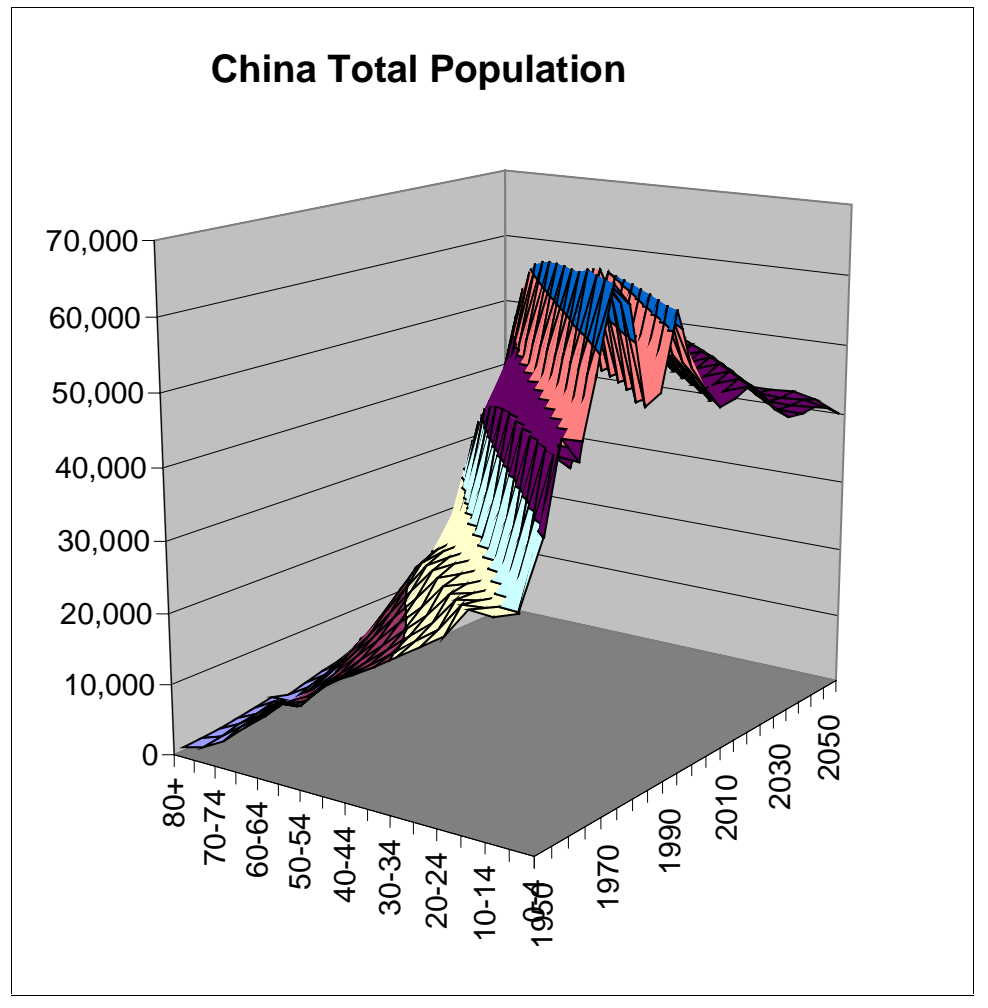

Figure 1a

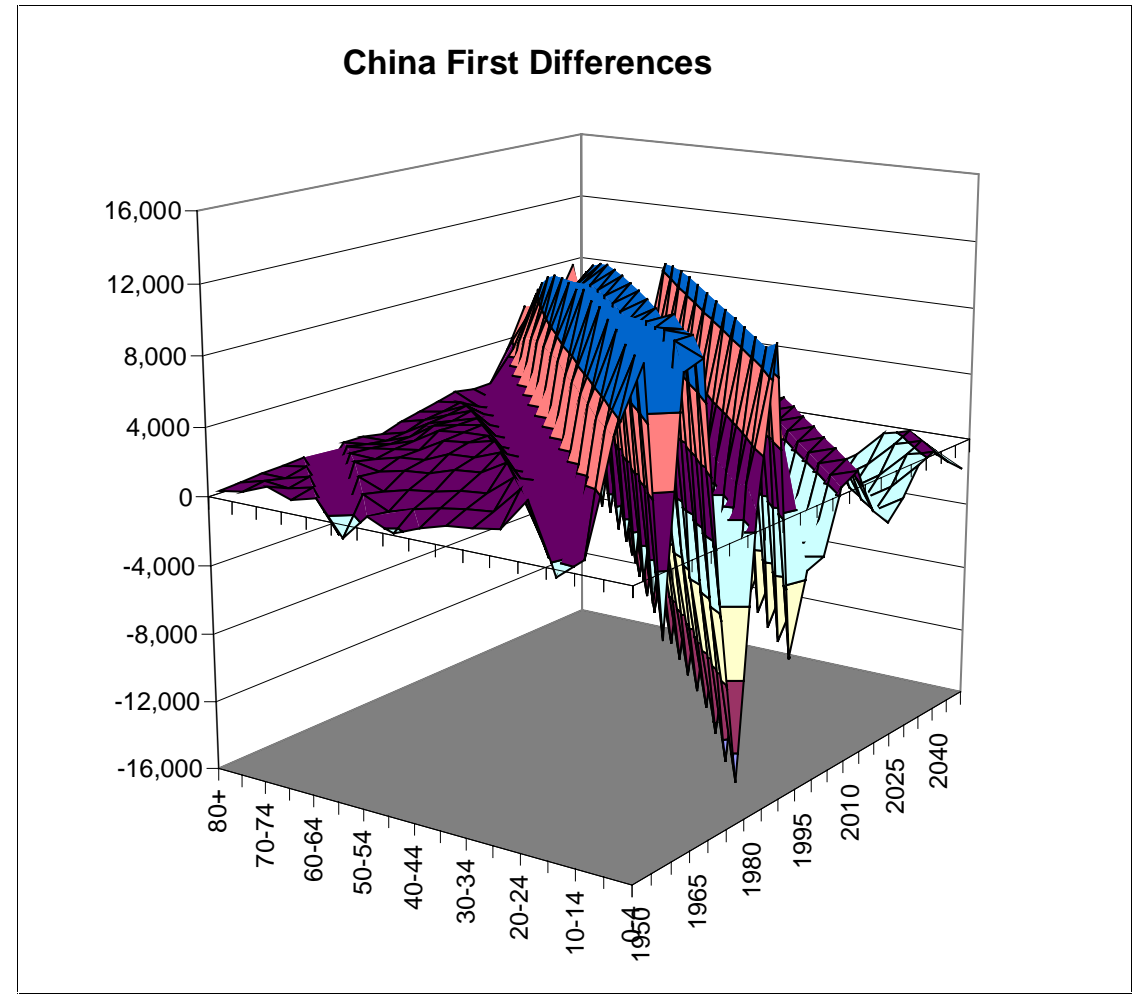

Figure $1 b$ 


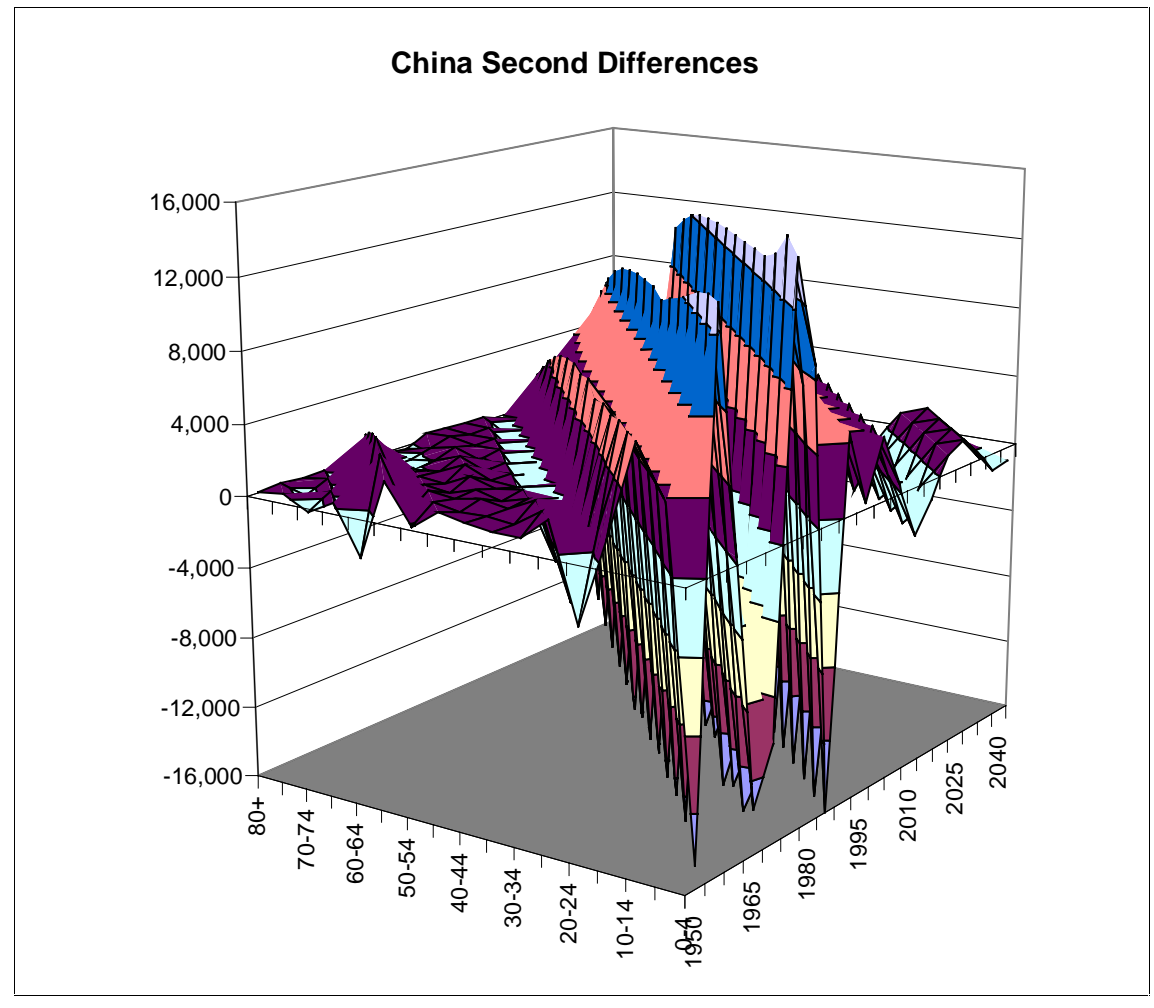

Figure 1c

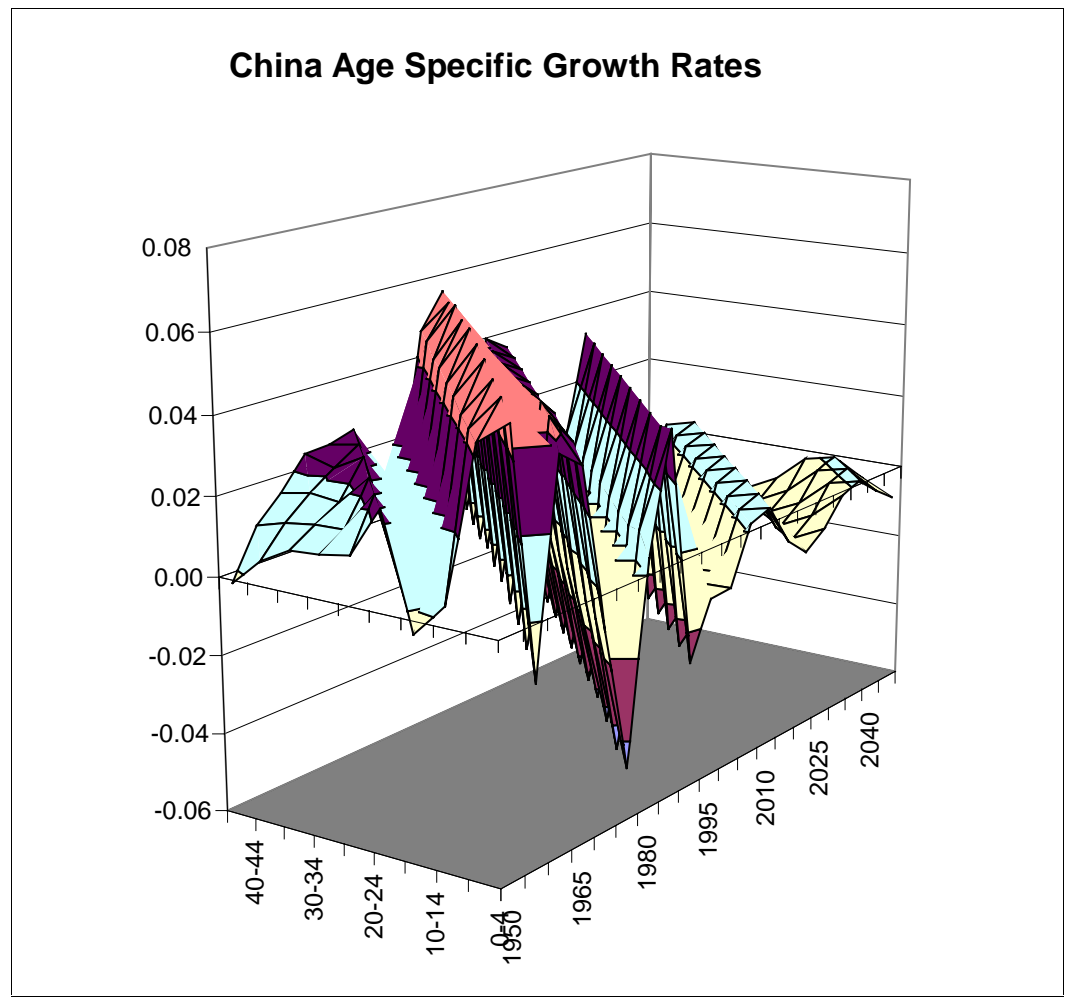

Figure 1d 


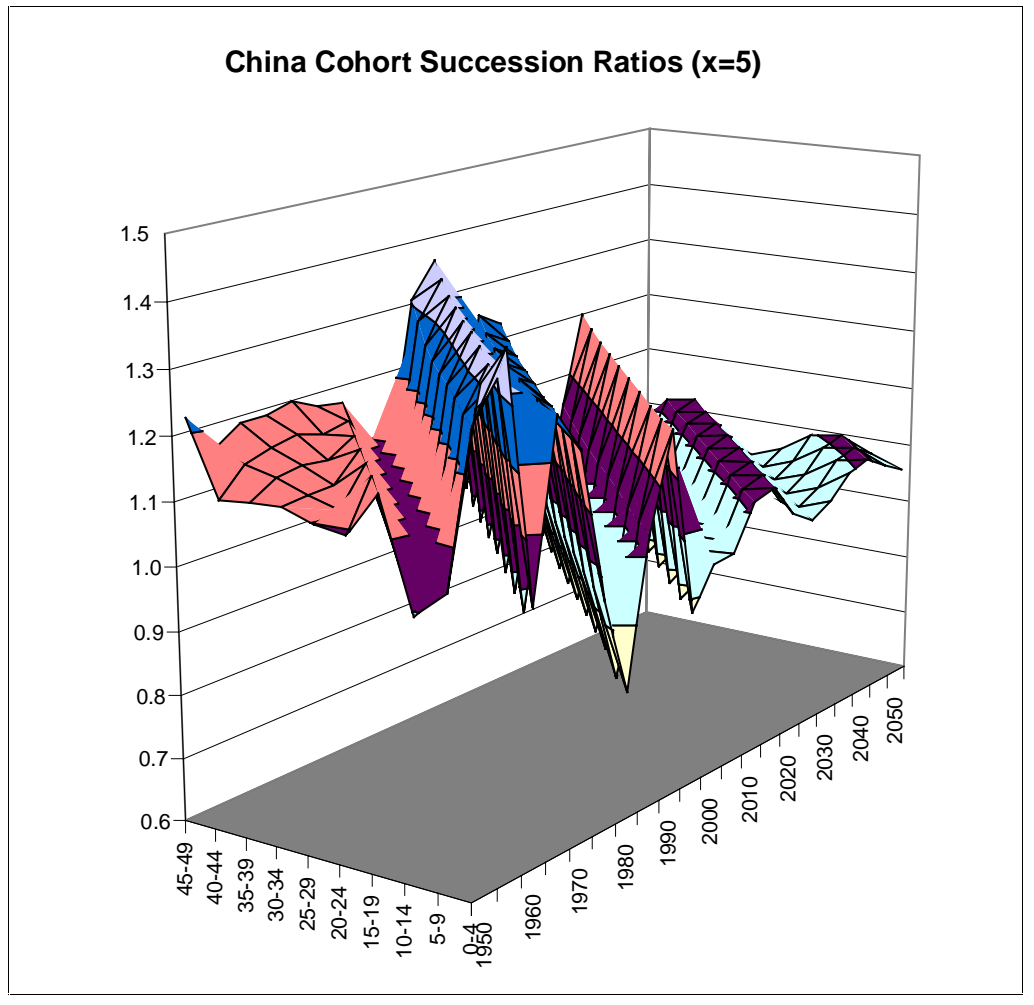

Figure 1e

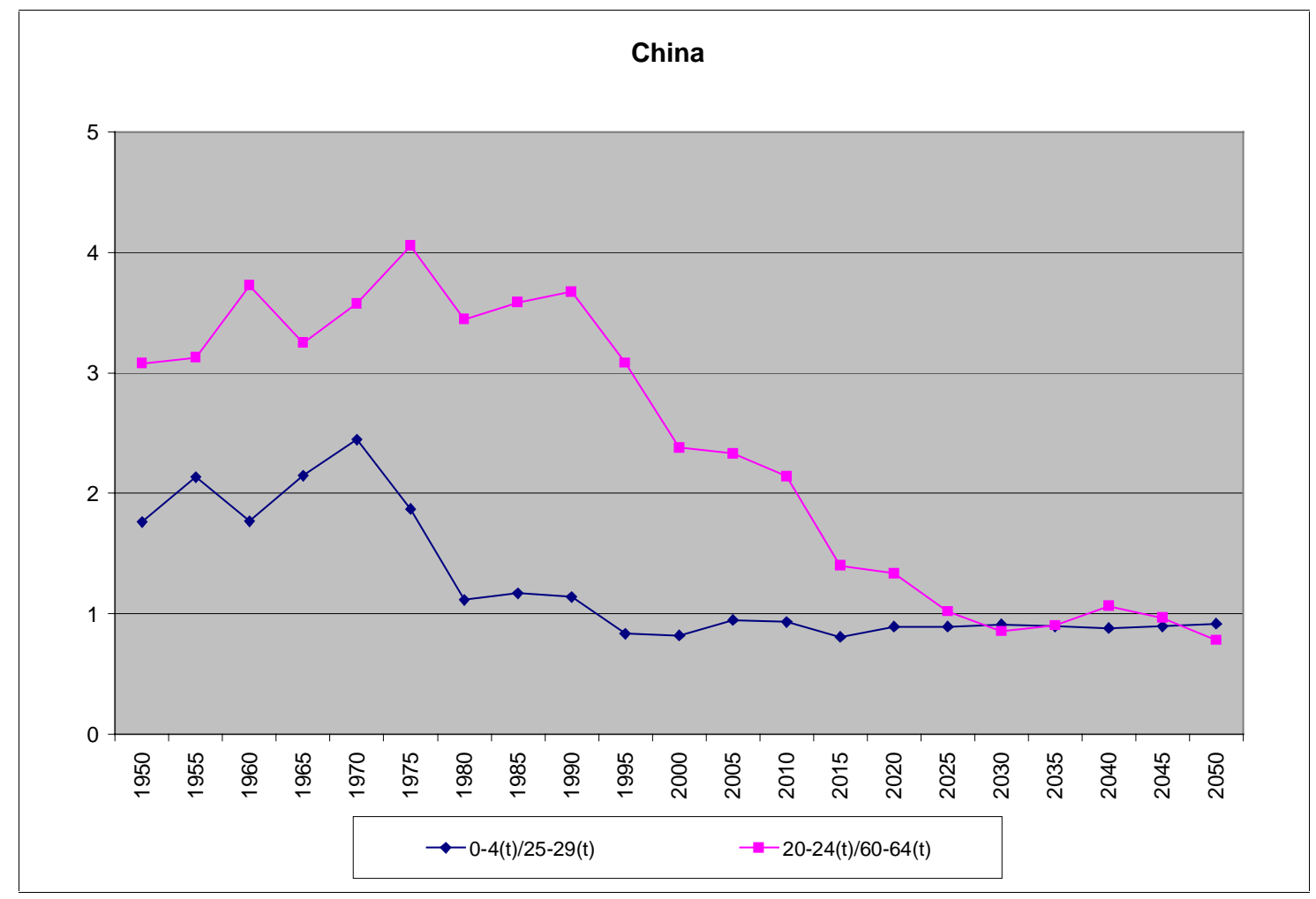

Figure 2 


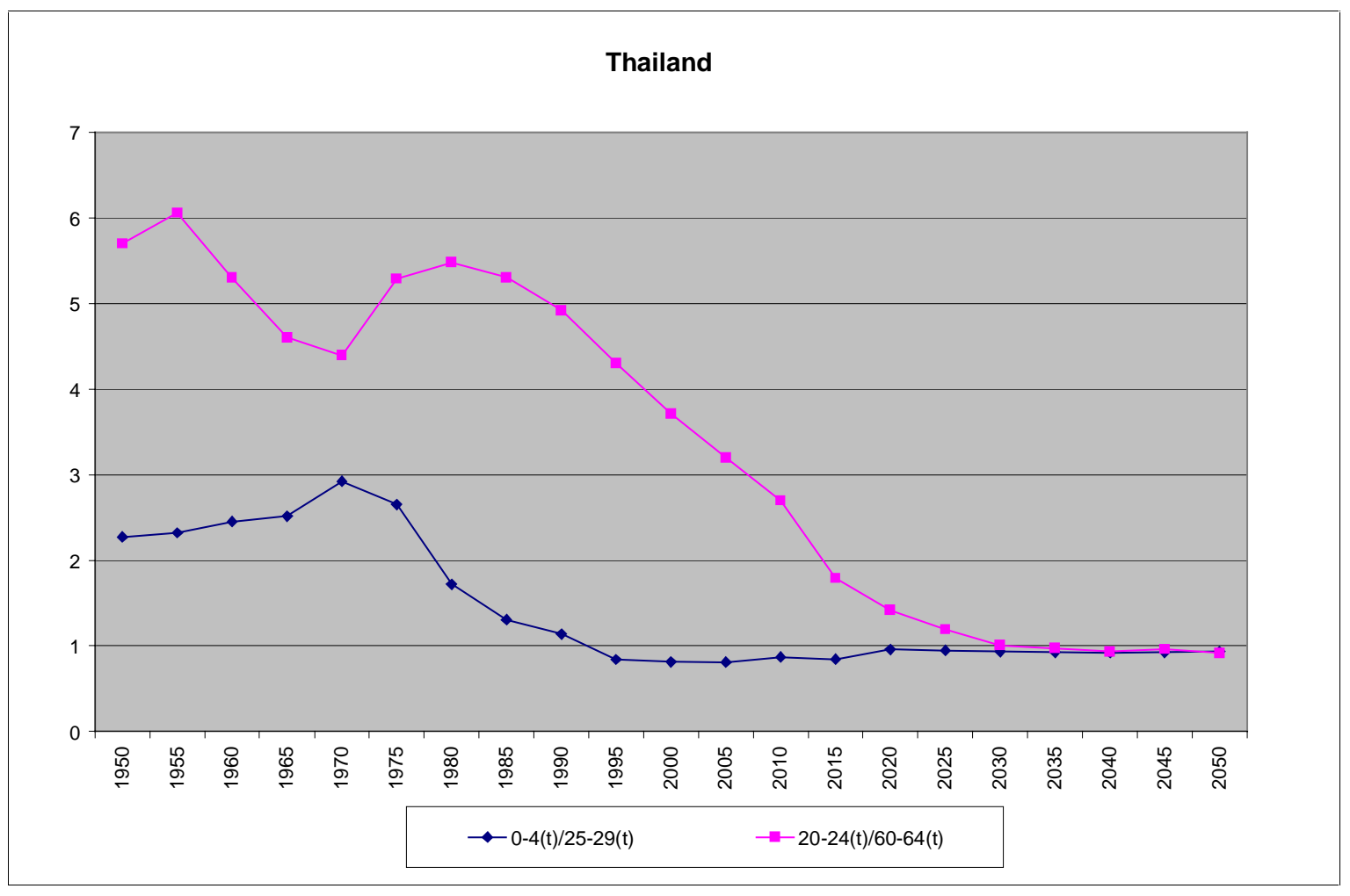

Figure 3

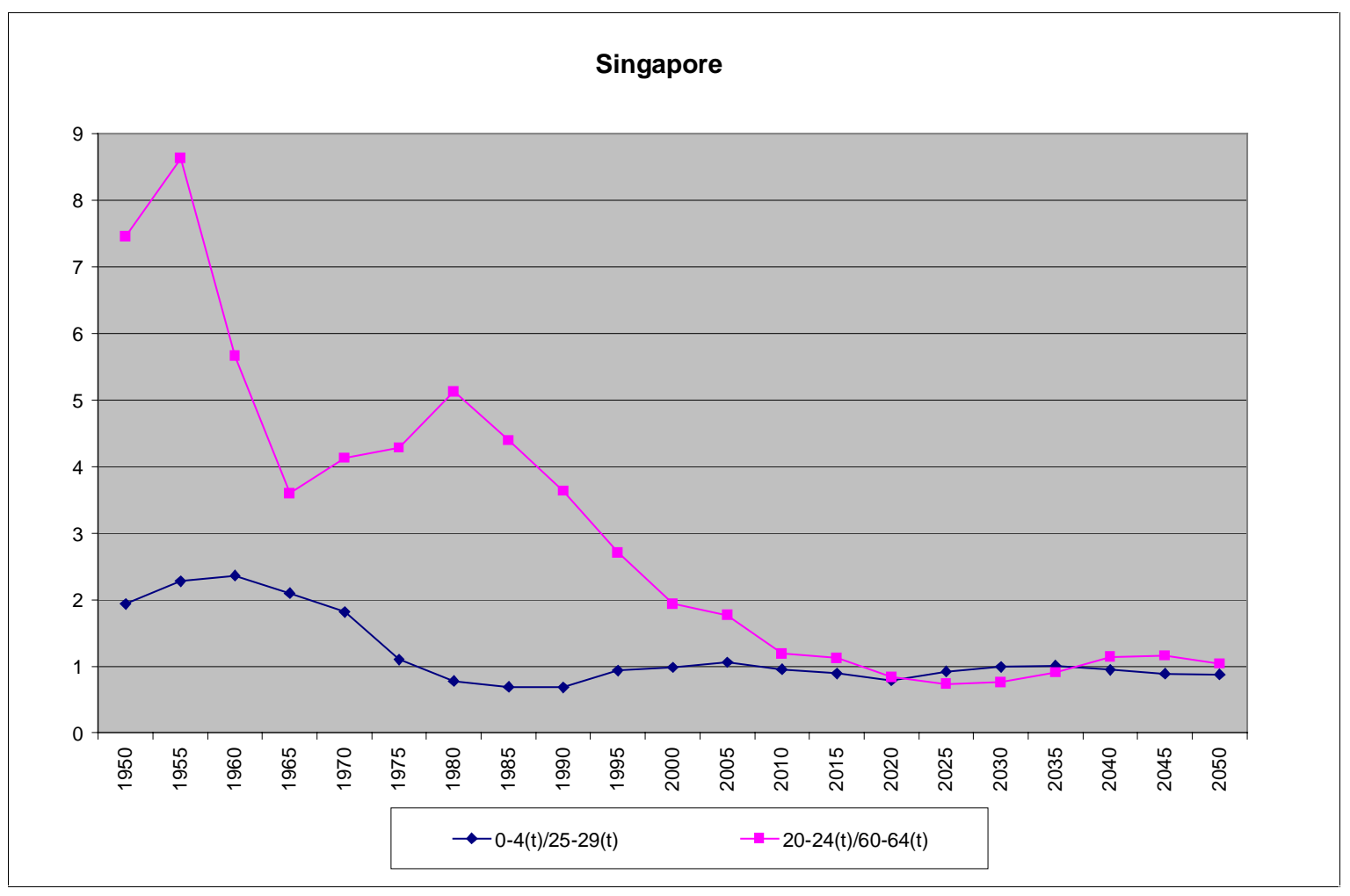

Figure 4 
We have to do more to move this analysis toward incorporating the aspect of human capital formation. This is important for answering the question whether, e.g., a labor force replacement ratio below 1.0 is something to be concerned about. Since educational attainment of the labor force is a most important determinant of future productivity and hence impacts on the future standard of living and intergenerational equity, we will have to find a way to include it into these calculations.

Table 1 gives some very simple calculations based on the recently produced projections of educational attainment for 13 world regions (Lutz and Goujon 2001). These first global forecasts of the population by educational attainment distinguish between four educational groups: no education, some primary, some secondary and some tertiary. For the purpose of simple calculation, we combine them into two groups and simply assume that those aged 20-65 with no or some primary education have a productivity of 1.0, and those aged 20-65 with some secondary or higher education have a productivity of 2.0. Those below age 20 and above age 65 are assumed to be not productive in this model. Table 1 presents a ratio calculated in the following way: The number of working age (20-65) men and women with higher education is multiplied by the factor of 2.0 and added to the working age population with lower education. This sum is then divided by the total population (all ages) in the given year. This ratio can be interpreted as a measure of the age- and education-dependent productivity of given populations.

Table 1. Change in age- and education-dependent productivity for selected world regions. Men and women aged 20-65 with no and some primary education have a productivity of 1.0; those with secondary and tertiary education, 2.0. Based on IIASA's educational projections (in millions).

\begin{tabular}{l|rrrr|rrrr}
\hline Region & \multicolumn{7}{|c|}{2000} & \multicolumn{3}{|c}{2030} \\
& $(1)$ & $(2)$ & $(3)$ & $(4)^{*}$ & $(1)$ & $(2)$ & $(3)$ & $(4)^{*}$ \\
& Low & High ed & Pop & Ratio & Low & High ed & Pop & Ratio \\
ed & & & & & & \\
South Asia & 498 & 191 & 1367 & $\mathbf{0 . 6 4}$ & 731 & 492 & 2030 & $\mathbf{0 . 8 4}$ \\
China Region & 449 & 391 & 1408 & $\mathbf{0 . 8 7}$ & 274 & 749 & 1617 & $\mathbf{1 . 1 0}$ \\
Pacific Asia & 169 & 94 & 476 & $\mathbf{0 . 7 5}$ & 179 & 218 & 605 & $\mathbf{1 . 0 2}$ \\
Western & 75 & 201 & 456 & $\mathbf{1 . 0 5}$ & 25 & 247 & 480 & $\mathbf{1 . 0 8}$ \\
Europe & & & & & & & & \\
North & 9 & 177 & 314 & $\mathbf{1 . 1 6}$ & 15 & 199 & 390 & $\mathbf{1 . 0 6}$ \\
America & & & & & & & & \\
\hline
\end{tabular}

$*(4)=[(1)+2 \times(2)] /(3)$ 
Table 1 presents these calculations for five selected world regions. South Asia has the lowest ratio in 2000 and is likely to have the lowest one in 2030, despite some improvements. The main reason for this poor performance is the low educational level of the total population combined with still high youth dependency. The China region, which has a comparable total population size in 2000, has much better starting conditions in 2000 (even better than South Asia will have in 2030) due to strong past investments in education and lower fertility. By 2030 China is likely to reach a high level of 1.10 which, according to our specifications, will be higher than the age and education-dependent productivity ratios for Western Europe and North America (of course, these measures do not give special attention to highly-advanced technological skills). But these trends clearly indicate that China will strongly gain in terms of international competitiveness. The region of Pacific Asia, which includes most of Southeast Asia, is also set for an impressive improvement. While being between China and South Asia in 2000, it is likely to have even more impressive gains in age-and education-dependent productivity than China.

But even this weighting by education does not yet bring us to a full operationalization of intergenerational equity concerns in the context of "population balance." To make further progress in this direction, for the time being we will leave the realm of empirical data and enter the world of simulation.

\section{A First Attempt to Simulate Age, Cohort and Human Capital Aspects of "Population Balance"}

Population balance can mean many things. For example, it could mean population in balance with its environment. It could mean a balance of sex or age groups. It could mean a population whose growth is in balance with the growth of the capital stock and infrastructure that it needs for its production. But it would be difficult to understand "population balance" if we were to integrate them all into a single very complex framework. Instead, we go to the other extreme of producing a simple model of population in balance between its period aspect (production) and its cohort aspect (lifetime consumption). By studying first this aspect of population balance and later adding other elements to it, we hope eventually to produce a rich understanding of "population balance."

Population in balance with its environment is commonly discussed under the rubric of "sustainable development." In that context, the definition of balance is clear. Generations that come first are not to consume and produce in such a way that future generations are denied the possibilities that the earlier generations possessed. More briefly, balance is intergenerational equity. Because of the importance of intergenerational equity itself and because we anticipate soon adding environmental balance to our framework, we interpret balance here to mean intergenerational equity.

We assume a one-sex population closed to migration and with fixed age-specific mortality rates. There are two types of people: those with education and those without education. Two things are allowed to vary exogenously over time: age-specific fertility rates, summarized by the total fertility rate (TFR), and probability of a young person becoming educated. 
The life cycle is assumed to have three stages. In the first, children consume, either acquire education or not, but do not produce. In the second, adults consume and produce both children and output. In the third, retirees consume, but again do not produce output. Output in any year depends on the numbers of educated and uneducated people in the second stage weighted by their relative productivities.

$Y(t)=N(2, t, u) \cdot \pi(u)+N(2, t, e) \cdot \pi(e)$,

where $N(2, t, u)$ is the number of uneducated people in life cycle stage 2 in year $t, \pi(u)$ is the productivity of unskilled workers, and $N(2, t, e)$ and $\pi(e)$ have the analogous interpretations for educated workers.

People aged 6 and above in stage 1 can obtain one year of education every year. Once enrolled in education at age 6, they remain in school or other training to the end of the stage. The enrollment rate at age 6 changes exogenously over time. Each year of education is assumed to cost $E$ euros per year. The total cost of education in year $t$ is simply the product of the education cost and the number of children in school.

$$
\operatorname{EDCOST}(t)=N(1, t, e) \cdot E
$$

where $N(1, t, e)$ is the number of people in stage 1 who are in school and $E$ is the per annum cost of schooling.

Output is divided among the people alive at time $t$. People in stage 1 get to consume a fraction, $w(1)$ of the amount allocated to people in stage 2. People in stage 3 get to consume a fraction $w(3)$ of the amount allocated to people in stage 2 . The two fractions are considered exogenous here.

The income of a person in stage 2 in year $t$, then, is just $I(2, t)$, where

$$
I(2, t)=\frac{Y(t)-E D C \operatorname{COST}(t)}{w(1) \cdot(N(1, t, u)+N(1, t, e))+(N(2, t, u)+N(2, t, e))+w(3) \cdot(N(3, t, u)+N(3, t, e))} .
$$

The income of a person in stage 1 in year $t$ is

$$
\begin{aligned}
& I(1, t)=w(1) \cdot I(2, t), \text { and } \\
& I(3, t)=w(3) \cdot I(2, t) .
\end{aligned}
$$

The lifetime average income of a person born in year $c$ who survives to his/her $100^{\text {th }}$ birthday is:

$$
\operatorname{LIFE}(c)=\left[\prod_{a=0}^{a 1} I(1, a+c) \cdot \prod_{a=a 1+1}^{a 2} I(2, a+c) \cdot \prod_{a=a 2+1}^{99} I(3, a+c)\right]^{1 / 100} .
$$

Before we look at simulation results from this very simple model, it is perhaps important to strongly emphasize that we do not interpret this to be a realistic model of population and production. To take the model in the direction of greater realism, we would have to incorporate factors such as the capital stock so that we could capture the capital dilution effect of faster economic growth, the environment, so that we could capture diminishing returns generated by fixed resources, endogenous technological change, so that we could incorporate the effects of scale and education on the rates of invention and adoption of new technologies. All of these would greatly complicate the 
model and distract us from the main point that we wish to make here. In the simplest imaginable age-structured model of population, which takes into account both its period aspect (production) and its cohort aspect (lifetime consumption), balance is best.

This model differs from standard economic models by having no discount rates. The logic of balance in the sense of sustainable development requires that all cohorts be treated equally; this rules out discounting across cohorts. Discounting within cohorts is a different matter. Instead of Eq. (4), we could have chosen to look at expected lifetime income, where the expectation was taken over the probabilities of surviving to different ages. Given that we used a life expectancy of birth of 70 years and that it does not vary over the simulations, the results would not have been much different. We have tried here to be as simple and transparent as possible and did not want to introduce any unnecessary complications.

Figures 5 through 9 show simulations of the simple model under the following assumptions:

1. Stage 1 lasts from age 0 through age 19. Education and training lasts from age 6 through age 19. Stage 2 lasts from age 20 through age 64, and stage 3 lasts from age 65 through 99.

2. Simulations were run for 200 years, resulting in complete lifetime observations on 100 cohorts.

3. All simulations are begun from stable population age distributions.

4. $\pi(u)=100$ and $\pi(e)=200$.

5. $\mathrm{E}=100$.

6. $\quad w(1)=0.4$ and $w(3)=0.9$.

7. Age-specific mortality rates are taken from the Coale-Demeny model, West life table for females with life expectancy at birth equal to 70 .

8. The pattern of age-specific fertility rates is that given by the U.S. Bureau of the Census for Argentina in 2000.

Preliminary sensitivity tests shows that the results concerning population balance that we see below are robust to plausible changes in these assumptions.

Figure 5 shows the lifetime incomes of stable populations for three levels of proportion educated for total fertility rates ranging from 0.01 to 6.0. Given the mortality rates that were used, replacement fertility occurs at a total fertility rate of $2.13 .^{2}$ The first thing to notice about this graph is that lifetime income in stable populations has a single interior peak in the range of relevant total fertility rates. Total fertility rates, and therefore population growth rates, that are too high lead to lower average lifetime incomes. Total fertility rates that are too low, and therefore lead to rapid population shrinkage, also cause lower average lifetime incomes.

The shapes of the curves in Figure 5 are the result of two opposing forces. With the mortality rates used here, higher total fertility rates cause the fraction of the stable

\footnotetext{
${ }^{2}$ For ease of comprehension, total fertility rates are transformed into their usual metric for a two-sex population.
} 
population in stage 2 to decline. This, along with the increase in the population in stage 1 , causes the numerator of Eq. (3a) to decrease. This is offset to some extent by the age structure effects at stages 1 and 3. According to our assumptions, the population in stage 1 has a lower command over income than the population in stage 3. Higher total fertility rates, therefore, also cause the denominator of Eq. (3a) to decrease.

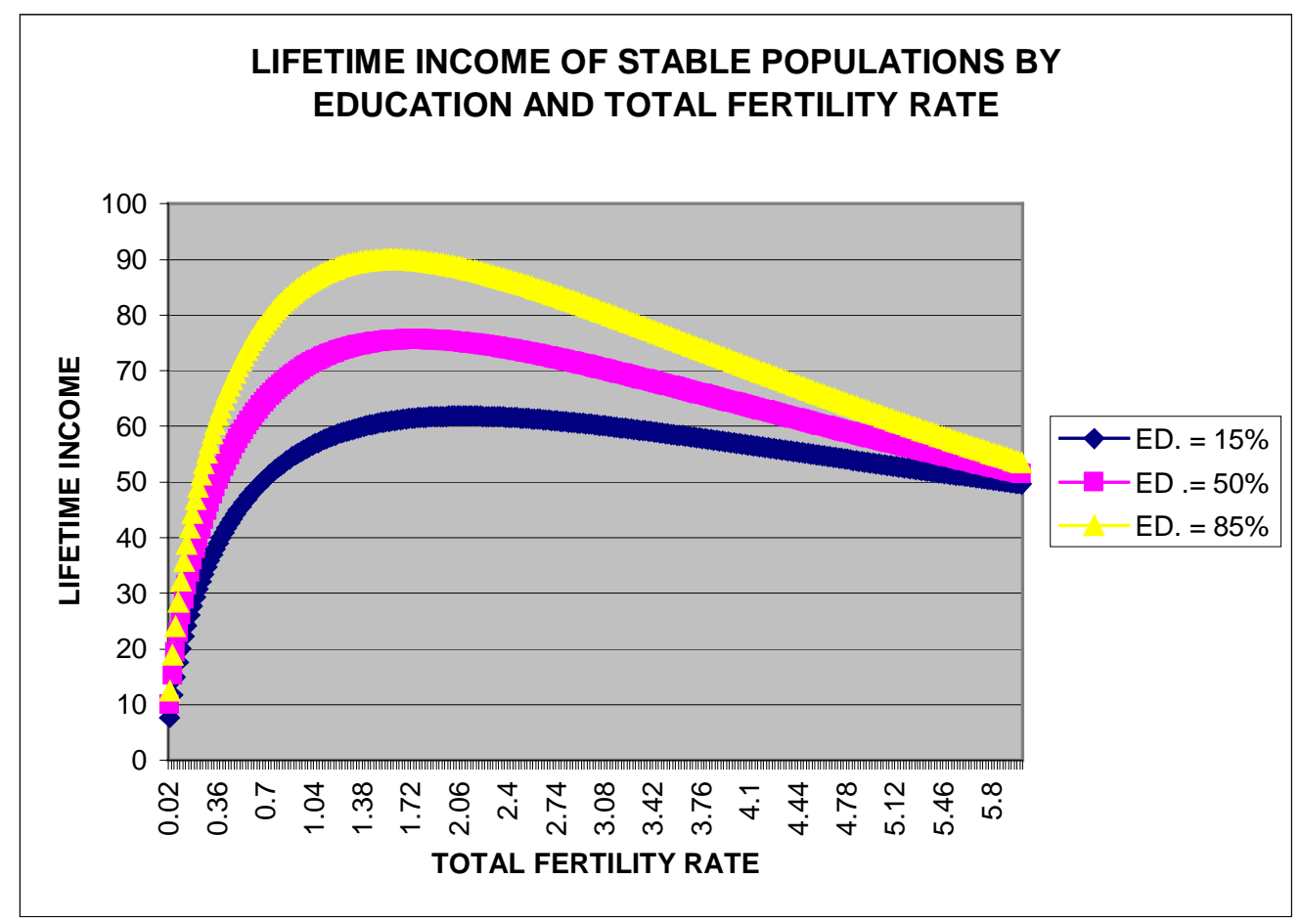

Figure 5

Figure 5 also shows that the peak level of lifetime income occurs at lower total fertility rates for more educated than for less educated populations. This happens because educating children is a costly process that must occur prior to recouping its benefits. Age structures with lower proportions in stage 1 are therefore somewhat more advantageous.

In Figure 6 we have plotted average lifetime incomes in stable populations for three levels of fertility and for proportions educated between zero and one. Regardless of the level of education, lifetime income is always higher when the total fertility rate is at replacement than when it is either 1.0 or 3.0. This is just another way of seeing the advantage of population balance.

In Figure 7 we can see the effects of an education transition on the lifetime income of people in three different stable populations. The figure shows the average lifetime incomes of the 100 cohorts for which we have a complete 100 years of observation (i.e., simulation). ${ }^{3}$ The education transition begins in year 100, when 85 percent of the children at age 6 enter school instead of the earlier 15 percent. The three

\footnotetext{
${ }^{3}$ Cohort 1 lives from year 1 through year 100; cohort 2 lives from year 2 to year 101, and so on.
} 
cases are again a stationary population associated with replacement level fertility, a growing population with a TFR of 3.0 and a shrinking population with a TFR of 1.0.

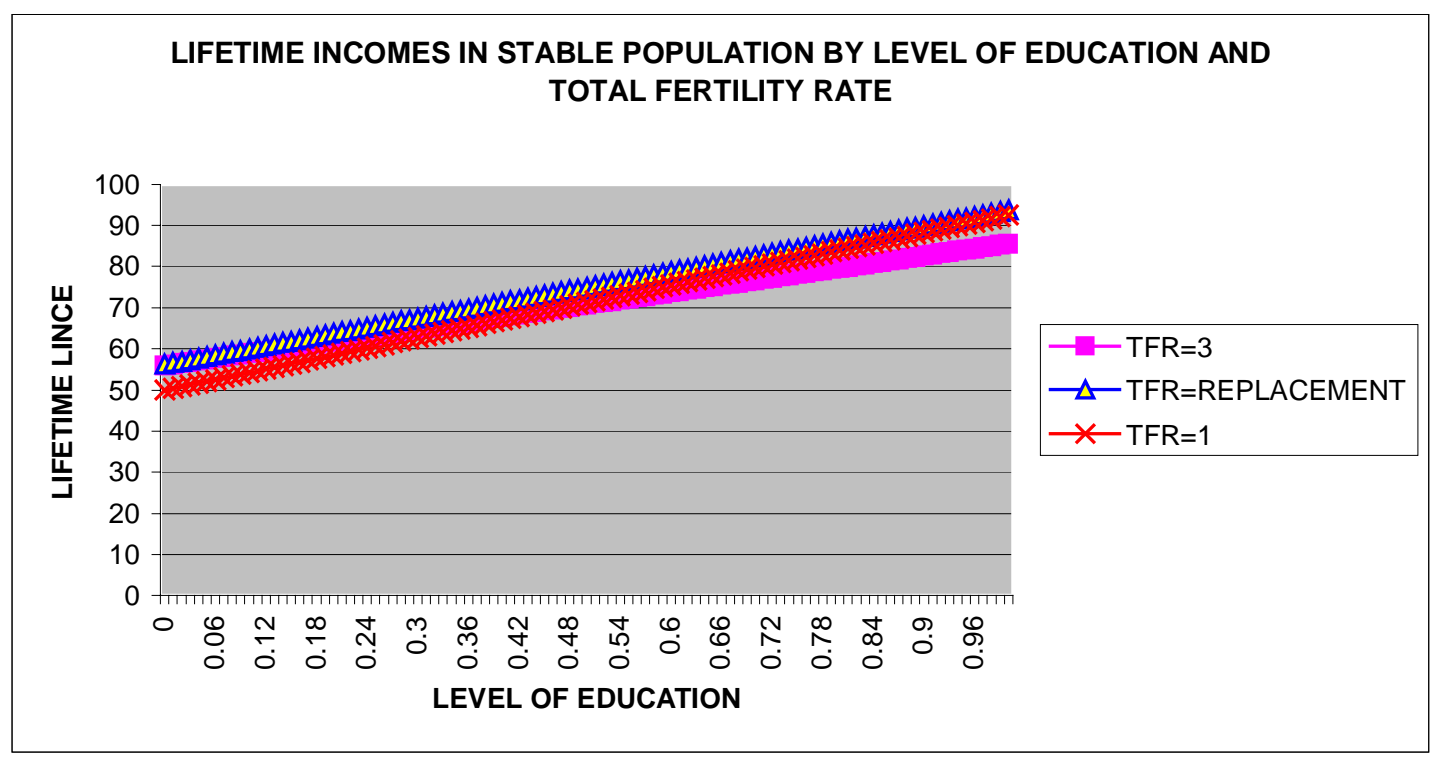

Figure 6

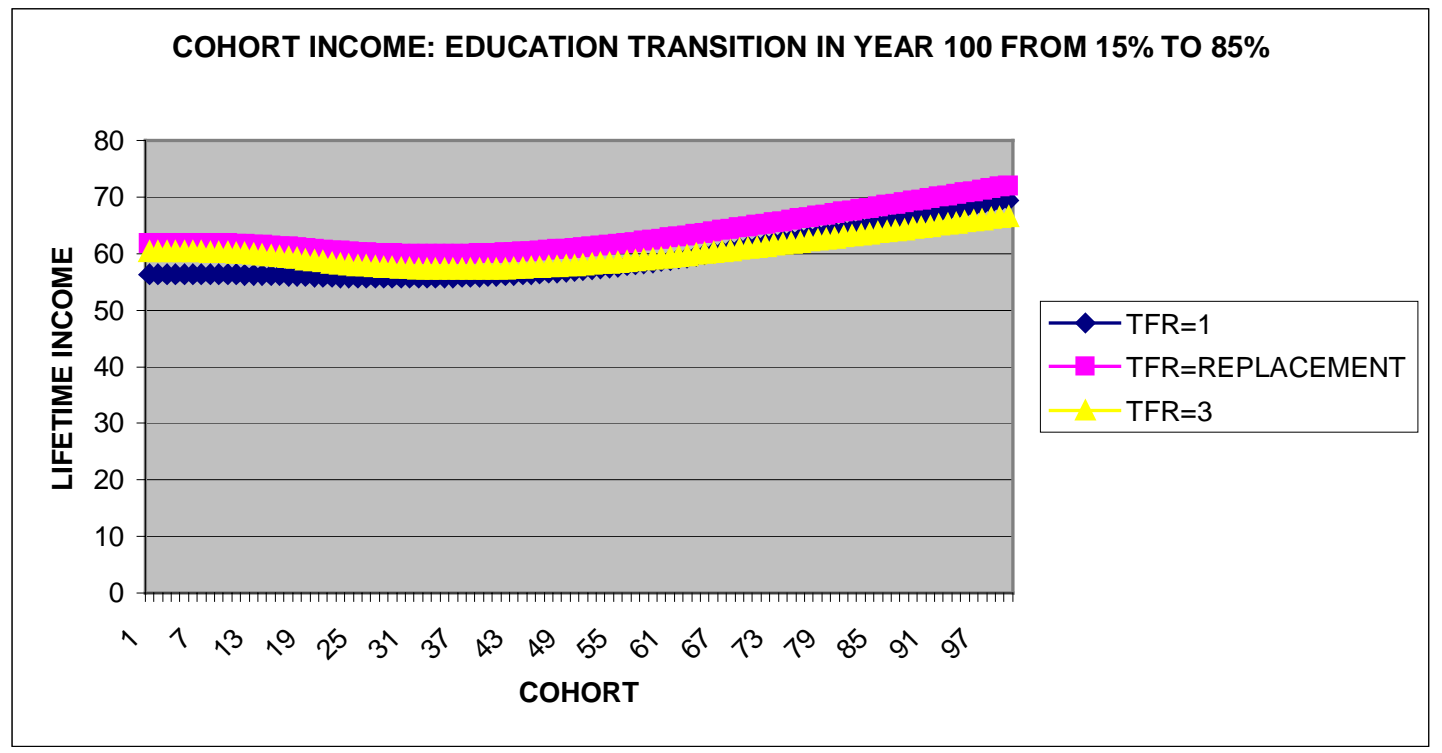

Figure 7

The curves in Figure 7 fall and then rise. They fall because people in the early cohorts pay for part of the increased schooling costs when they are elderly and do not live long enough to enjoy any of the increased productivity of the educated children. It rises because eventually the increased productivity of the educated dominates the earlier educational investment costs. Again, we see that balance is best. Lifetime incomes are always higher in the population with replacement level fertility. 
Figure 8 is like Figure 7 except that here we look at the effects of a fertility transition from a TFR of 4.0 to a TFR of 2.0 occurring in year 100 in the context of three different levels of education. As we would expect from what we have seen before, these fertility transitions increase average lifetime income at each level of education.

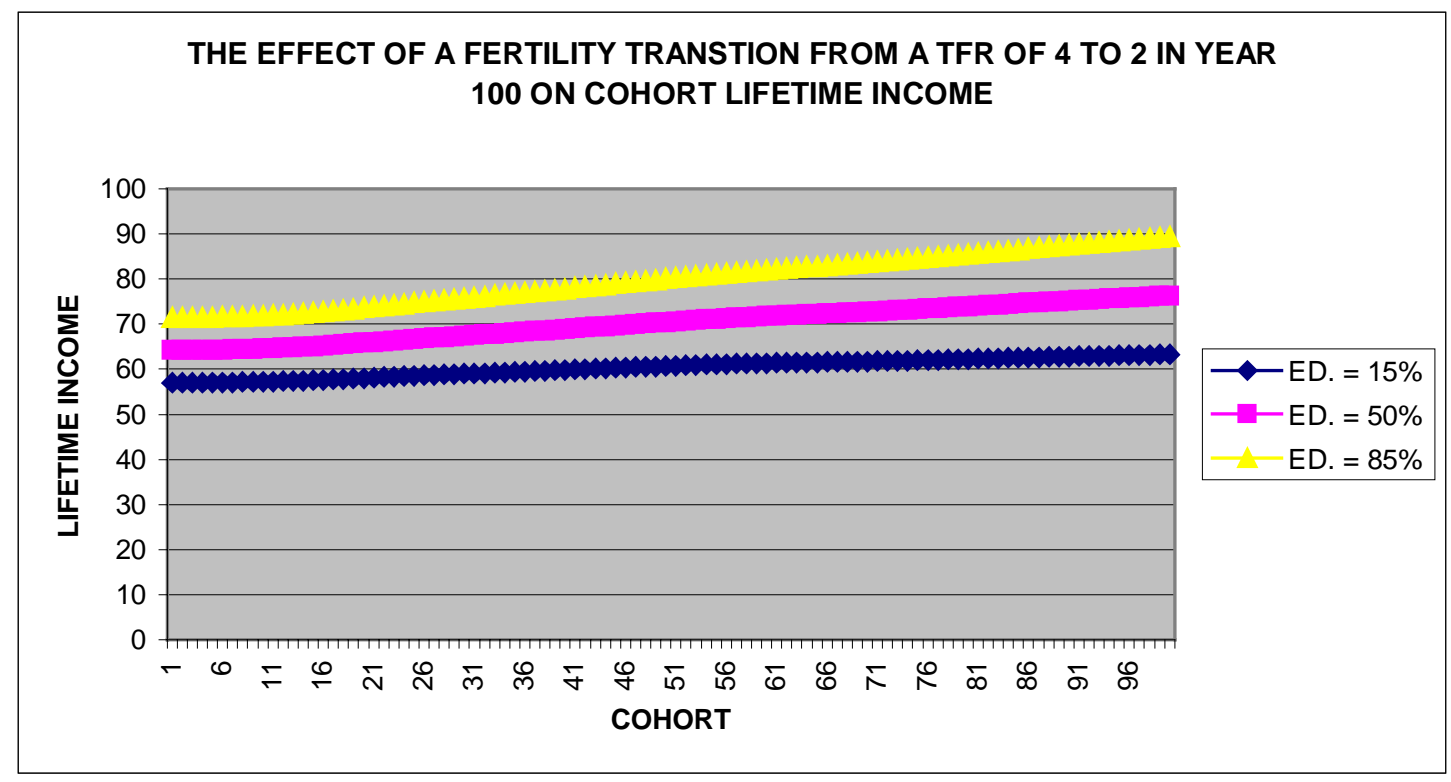

Figure 8

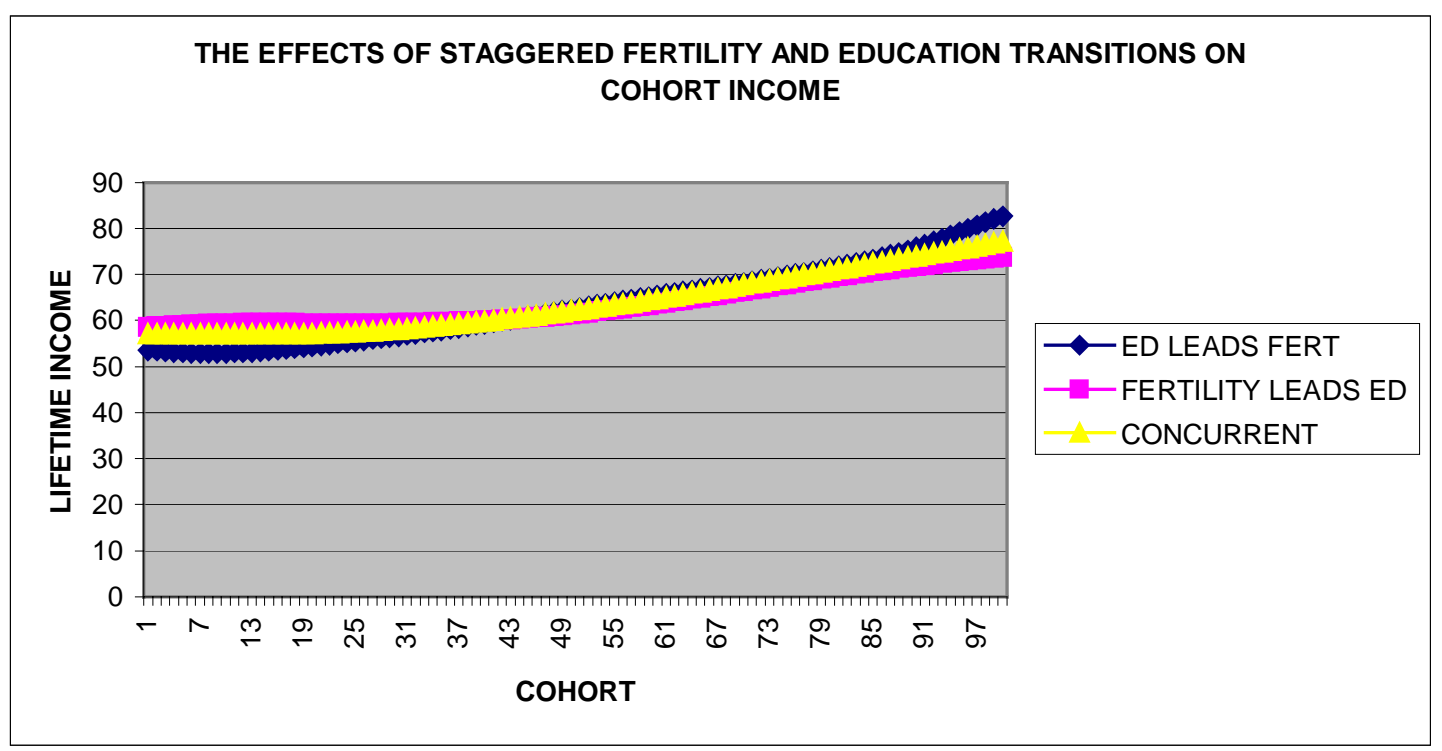

Figure 9

Figure 9 combines the situations of Figures 7 and 8 and looks at the joint fertility and education transitions. All simulations between with the stable age distribution are associated with a TFR of 4.0. We consider three cases. In one the education transition (an increase in the percent educated from 15 percent to 85 percent) precedes the fertility 
decrease (a decrease in the TFR from 4.0 to 2.0). The education transition begins in year 75 and the fertility transition begins 25 years later. In the second case, the timing is reversed and the fertility transition begins in year 75 and the education transition follows 25 years later. In the third case, the transitions are concurrent, both beginning in year 88 .

In the case where the education transition leads the fertility transition, lifetime income of the earlier cohorts is suppressed, and lifetime income of the later cohorts is enhanced relative to the situation where the timing is reversed. If we were to consider more cohorts, however, eventually the three lines would coincide as the stable age structures become identical. We could have produced the analogs of Figures 8 and 9 for transitions from a TFR of 2.0 to a TFR of 1.0. Regardless of the timing of the transitions, lifetime incomes would fall.

We have shown that population balance appears in the simplest possible agestructured model of consumption and production. The problems of rapid population growth and rapid population shrinkage are indeed two sides of the same coin.

\section{Outlook}

This paper has presented a first and rather scattered attempt in defining a new concept tentatively called "population balance." It first discusses the desirability of such a concept bridging the population growth and population aging concerns by giving explicit consideration to the implications of age-structural changes and by endogenizing human capital formation. In this paper the key criterium of sustainability, namely intergenerational equity, is being discussed and simulated in the context of comparing lifetime income across cohorts. A next step will be to expand the model to explicitly incorporate environmental constraints and feedbacks.

\section{References}

Horiuchi, Shiro and Samuel H. Preston. 1988. Age-specific growth rates: The legacy of past population dynamics. Demography 25(3):429-441.

Keyfitz, Nathan. 1990. The profile of intercohort increase. Mathematical Population Studies 2(2):105-117.

Keyfitz, Nathan. 1988. The Asian road to democracy. Society 26(1):71-76.

Keyfitz, Nathan. 1987. The demographic discontinuity of the 1940s. Working Paper WP-87-92. Laxenburg, Austria: International Institute for Applied Systems Analysis.

Lutz, Wolfgang, Anne Goujon, and Gabriele Doblhammer-Reiter. 1999. Demographic dimensions in forecasting: Adding education to age and sex. Pages 42-58 in W. Lutz, J.W. Vaupel, and D.A. Ahlburg (eds.), Frontiers of Population Forecasting. A Supplement to Vol. 24, 1998, Population and Development Review. New York: Population Council.

Lutz, Wolfgang and Anne Goujon. 2001. The world's changing human capital stock: Population forecasts by educational attainment. Forthcoming in Population and Development Review. New York: Population Council. 
Preston, Samuel H. and Ansley J. Coale. 1982. Age structure, growth, attrition and accession: A new synthesis. Population Index 48:217-259.

UN. 1999. World Population Prospects. The 1998 Revision. Volume II: Sex and Age. New York: United Nations. 